\title{
Erratum to: Insight into particle production mechanisms via angular correlations of identified particles in pp collisions at $\sqrt{s}=7 \mathrm{TeV}$
}

\author{
ALICE Collaboration ${ }^{\star}$ \\ CERN, 1211 Geneva 23, Switzerland
}

Received: 10 October 2019 / Accepted: 15 October 2019 / Published online: 10 December 2019

(C) CERN for the benefit of the ALICE collaboration 2019

Erratum to: Eur. Phys. J. C (2017) 77:569

https://doi.org/10.1140/epjc/s10052-017-5129-6

We have identified a mistake in how Fig. 1 is referenced in the text of the article Eur. Phys. J. C 77 (2017) no. 8, 569 which affected three paragraphs of the results section. The corrected three paragraphs as well as the unmodified accompanying figure are reproduced in this document with the correct labeling.

In addition, an editing issue led to a missing acknowledgements section. The missing section is reproduced at the end of this document in the manner in which it should have appeared in the published article.

\section{Amended paragraphs in the results section}

For correlation functions (a)-(h) in Fig. 1 the baseline reflecting the energy-momentum conservation is combined with several expected physics mechanisms. For same sign particle pairs of mesons (a)-(b), the near-side peak is consistent with the mini-jet mechanism combined with the Bose-Einstein correlations. The away-side ridge is also prominent, consistent with a mini-jet origin.

The particle-anti-particle correlations (e)-(h) also show a mini-jet like structure on the near-side and a weak awayside one. For pairs of non-identical particles Bose-Einstein and Fermi-Dirac effects are not present; however, resonances play a significant role in shaping the correlation function. Baryon and meson correlations are qualitatively similar. The only difference is the magnitude and width of the near-side peak, which is highest for kaons, lower for protons and lambdas, and lowest for pions. The shape and strength of the correlation functions (a distinct near-side peak) in (a)-(b) and (e)-(h) suggest that they might be dominated by significant mini-jet contributions.

In contrast to like-sign meson correlations, the baryonbaryon (combined with anti-baryon-anti-baryon) distributions for identical proton (c) and lambda (d) pairs show a qualitatively different effect of a wide near-side depression instead of the peak, combined with an away-side ridge. Such a structure resembles the one associated with the baseline global energy-momentum conservation. Thus, this strong near-side suppression means that the mechanisms which would produce a peak are either not present or produce a very different correlation shape. On the other hand, a clear correlation of particles with opposite baryon number (g)-(h) is observed, resembling the structures observed for unlikesign mesons. Based on the results of these studies we can draw the following conclusion: if we consider a process of mini-jet fragmentation as the one producing a strong, positive near-side correlation then baryon-anti-baryon pairs are produced in mini-jets (see (g)-(h)). However, producing more than one such a pair in a single fragmentation is strongly suppressed (see (c)-(d)).

The original article can be found online at https://doi.org/10.1140/ epjc/s10052-017-5129-6.

^e-mail: alice-publications@cern.ch 
Fig. 1 Correlation functions for identical-particle pairs: $\pi^{+} \pi^{+}+\pi^{-} \pi^{-}$, $\mathrm{K}^{+} \mathrm{K}^{+}+\mathrm{K}^{-} \mathrm{K}^{-}, \mathrm{pp}+\overline{\mathrm{pp}}$ $\Lambda \Lambda+\overline{\Lambda \Lambda}$ (left panel) and particle-anti-particle pairs: $\pi^{+} \pi^{-}, \mathrm{K}^{+} \mathrm{K}^{-}, \mathrm{p} \overline{\mathrm{p}}, \Lambda \bar{\Lambda}$ (right panel). Plots are mirrored around $\Delta \eta=0$
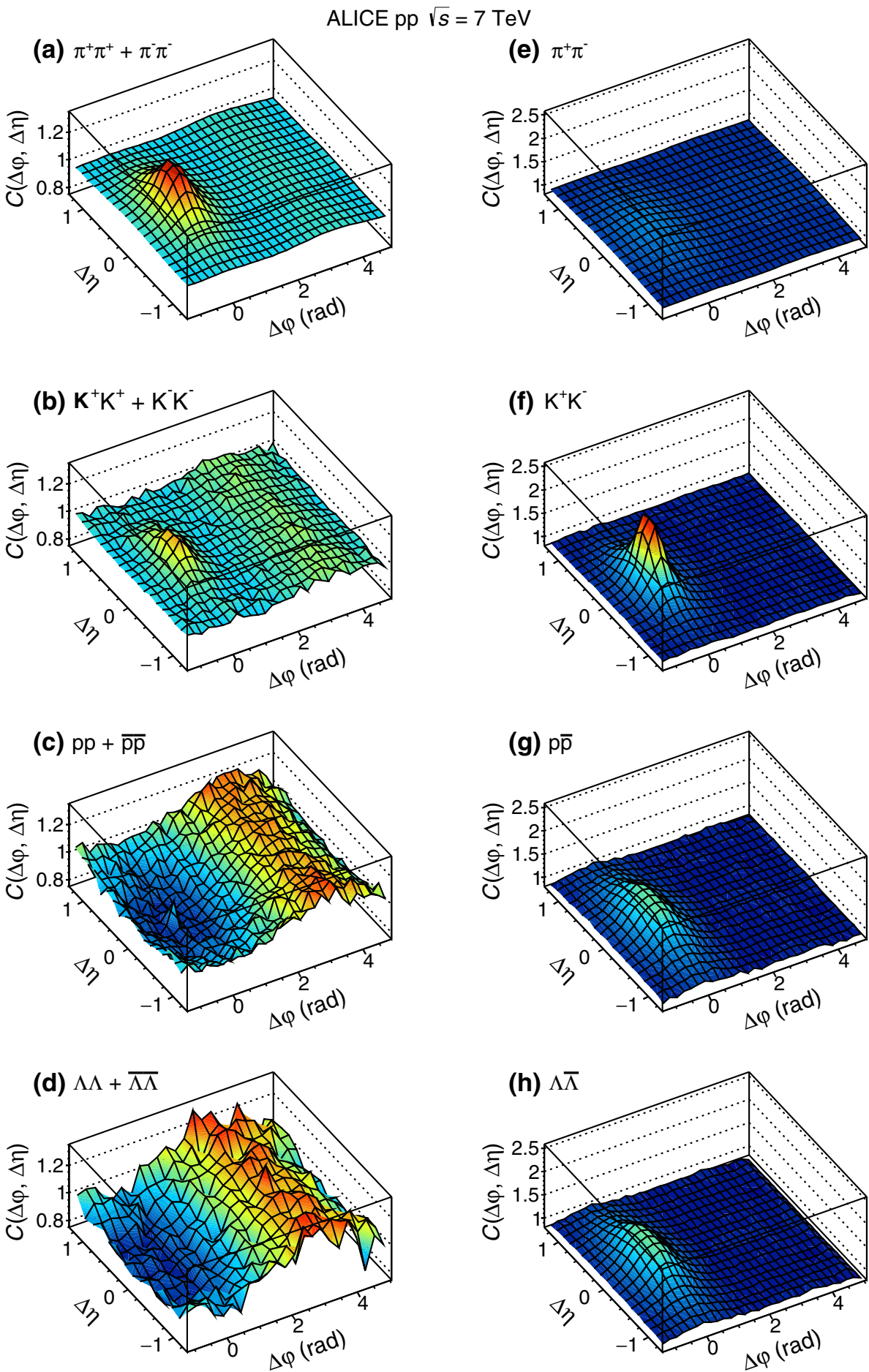

Acknowledgements The ALICE Collaboration would like to thank all its engineers and technicians for their invaluable contributions to the construction of the experiment and the CERN accelerator teams for the outstanding performance of the LHC complex. The ALICE Collaboration gratefully acknowledges the resources and support provided by all Grid centres and the Worldwide LHC Computing Grid (WLCG) collaboration. The ALICE Collaboration acknowledges the following funding agencies for their support in building and running the ALICE detector: A. I. Alikhanyan National Science Laboratory (Yerevan Physics Institute) Foundation (ANSL), State Committee of Science and World Federation of Scientists (WFS), Armenia; Austrian Academy of Sciences and
Nationalstiftung für Forschung, Technologie und Entwicklung, Austria; Conselho Nacional de Desenvolvimento Científico e Tecnológico (CNPq), Universidade Federal do Rio Grande do Sul (UFRGS), Financiadora de Estudos e Projetos (Finep) and Fundação de Amparo à Pesquisa do Estado de São Paulo (FAPESP), Brazil; Ministry of Science \& Technology of China (MSTC), National Natural Science Foundation of China (NSFC) and Ministry of Education of China (MOEC), China; Ministry of Science, Education and Sport and Croatian Science Foundation, Croatia; Ministry of Education, Youth and Sports of the Czech Republic, Czech Republic; The Danish Council for Independent Research I Natural Sciences, the Carlsberg Foundation and Danish 
National Research Foundation (DNRF), Denmark; Helsinki Institute of Physics (HIP), Finland; Commissariat à l'Energie Atomique (CEA) and Institut National de Physique Nucléaire et de Physique des Particules (IN2P3) and Centre National de la Recherche Scientifique (CNRS), France; Bundesministerium für Bildung, Wissenschaft, Forschung und Technologie (BMBF) and GSI Helmholtzzentrum für Schwerionenforschung $\mathrm{GmbH}$, Germany; Ministry of Education, Research and Religious Affairs, Greece; National Research, Development and Innovation Office, Hungary; Department of Atomic Energy Government of India (DAE) and Council of Scientific and Industrial Research (CSIR), New Delhi, India; Indonesian Institute of Science, Indonesia; Centro Fermi - Museo Storico della Fisica e Centro Studi e Ricerche Enrico Fermi and Istituto Nazionale di Fisica Nucleare (INFN), Italy; Institute for Innovative Science and Technology, Nagasaki Institute of Applied Science (IIST), Japan Society for the Promotion of Science (JSPS) KAKENHI and Japanese Ministry of Education, Culture, Sports, Science and Technology (MEXT), Japan; Consejo Nacional de Ciencia (CONACYT) y Tecnología, through Fondo de Cooperación Internacional en Ciencia y Tecnología (FONCICYT) and Dirección General de Asuntos del Personal Academico (DGAPA), Mexico; Nationaal instituut voor subatomaire fysica (Nikhef), Netherlands; The Research Council of Norway, Norway; Commission on Science and Technology for Sustainable Development in the South (COMSATS), Pakistan; Pontificia Universidad Católica del Perú, Peru; Ministry of Science and Higher Education and National Science Centre, Poland; Korea Institute of Science and Technology Information and National Research Foundation of Korea (NRF), Republic of Korea; Ministry of Education and Scientific Research, Institute of Atomic Physics and Romanian
National Agency for Science, Technology and Innovation, Romania; Joint Institute for Nuclear Research (JINR), Ministry of Education and Science of the Russian Federation and National Research Centre Kurchatov Institute, Russia; Ministry of Education, Science, Research and Sport of the Slovak Republic, Slovakia; National Research Foundation of South Africa, South Africa; Centro de Aplicaciones Tecnológicas y Desarrollo Nuclear (CEADEN), Cubaenergía, Cuba, Ministerio de Ciencia e Innovacion and Centro de Investigaciones Energéticas, Medioambientales y Tecnológicas (CIEMAT), Spain; Swedish Research Council (VR) and Knut \& Alice Wallenberg Foundation (KAW), Sweden; European Organization for Nuclear Research, Switzerland; National Science and Technology Development Agency (NSDTA), Suranaree University of Technology (SUT) and Office of the Higher Education Commission under NRU project of Thailand, Thailand; Turkish Atomic Energy Agency (TAEK), Turkey; National Academy of Sciences of Ukraine, Ukraine; Science and Technology Facilities Council (STFC), United Kingdom; National Science Foundation of the United States of America (NSF) and United States Department of Energy, Office of Nuclear Physics (DOE NP), United States of America.

Open Access This article is distributed under the terms of the Creative Commons Attribution 4.0 International License (http://creativecomm ons.org/licenses/by/4.0/), which permits unrestricted use, distribution, and reproduction in any medium, provided you give appropriate credit to the original author(s) and the source, provide a link to the Creative Commons license, and indicate if changes were made.

Funded by $\mathrm{SCOAP}^{3}$. 


\section{ALICE Collaboration}

J. Adam ${ }^{39}$, D. Adamová ${ }^{87}$, M. M. Aggarwal ${ }^{91}$, G. Aglieri Rinella ${ }^{35}$, M. Agnello ${ }^{31,113}$, N. Agrawal $^{48}$, Z. Ahammed ${ }^{137}$, S. Ahmad ${ }^{18}$, S. U. $A^{7 h n}{ }^{70}$, S. Aiola ${ }^{141}$, A. Akindinov ${ }^{55}$, S. N. Alam ${ }^{137}$, D. S. D. Albuquerque ${ }^{124}$, D. Aleksandrov ${ }^{83}$, B. Alessandro ${ }^{113}$, D. Alexandre ${ }^{104}$, R. Alfaro Molina ${ }^{65}$, A. Alici ${ }^{12,107}$, A. Alkin ${ }^{3}$, J. Alme ${ }^{22,37}$, T. Alt ${ }^{42}$, S. Altinpinar ${ }^{22}$, I. Altsybeev ${ }^{136}$, C. Alves Garcia Prado ${ }^{123}$, M. An ${ }^{7}$, C. Andrei ${ }^{81}$, H. A. Andrews ${ }^{104}$, A. Andronic ${ }^{100}$, V. Anguelov ${ }^{96}$, C. Anson ${ }^{90}$, T. Antičić ${ }^{101}, \quad$ F. Antinori ${ }^{110}$, P. Antonioli ${ }^{107}, \quad$ R. Anwar ${ }^{126}, \quad$ L. Aphecetche ${ }^{116}, \quad$ H. Appelshäuser ${ }^{61}$, S. Arcelli ${ }^{27}$, R. Arnaldi ${ }^{113}$, O. W. Arnold ${ }^{36,97}$, I. C. Arsene ${ }^{21}$, M. Arslandok ${ }^{61}$, B. Audurier ${ }^{116}$, A. Augustinus ${ }^{35}$, R. Averbeck ${ }^{100}$, M. D. Azmi ${ }^{18}$, A. Badalà ${ }^{109}$, Y. W. Baek ${ }^{69}$, S. Bagnasco ${ }^{113}$, R. Bailhache ${ }^{61}$, R. Bala ${ }^{93}$, A. Baldisseri ${ }^{15}$, R. C. Baral $^{58}$, A. M. Barbano ${ }^{26}$, R. Barbera ${ }^{28}$, F. Barile ${ }^{33}$, L. Barioglio ${ }^{26}$, G. G. Barnaföldi ${ }^{140}$, L. S. Barnby ${ }^{35,104}$, V. Barret $^{72}$, P. Bartalini ${ }^{7}$, K. Barth ${ }^{35}$, J. Bartke ${ }^{120, a}$, E. Bartsch ${ }^{61}$, M. Basile ${ }^{27}$, N. Bastid ${ }^{72}$, S. Basu ${ }^{137}$, B. Bathen ${ }^{62}$, G. Batigne ${ }^{116}$, A. Batista Camejo ${ }^{72}$, B. Batyunya ${ }^{68}$, P. C. Batzing ${ }^{21}$, I. G. Bearden ${ }^{84}, \quad$ H. Beck ${ }^{96}, \quad$ C. Bedda ${ }^{31}$, N. K. Behera ${ }^{51}$ ， I. Belikov ${ }^{66}$ ， F. Bellini27, H. Bello Martinez ${ }^{2}$, R. Bellwied ${ }^{126}$, L. G. E. Beltran ${ }^{122}$, V. Belyaev ${ }^{77}$, G. Bencedi ${ }^{140}$, S. Beole ${ }^{26}$, A. Bercuci ${ }^{81}$, Y. Berdnikov ${ }^{89}$, D. Berenyi ${ }^{140}$, R. A. Bertens ${ }^{54,129}$, D. Berzano ${ }^{35}$, L. Betev ${ }^{35}$, A. Bhasin 93 , I. R. Bhat ${ }^{93}$, A. K. Bhati ${ }^{91}$, B. Bhattacharjee ${ }^{44}$, J. Bhom ${ }^{120}$, L. Bianchi ${ }^{126}$, N. Bianchi ${ }^{74}$, C. Bianchin ${ }^{139}$, J. Bielčík ${ }^{39}$, J. Bielčíková ${ }^{87}$, A. Bilandzic ${ }^{36,97}$, G. Biro ${ }^{140}$, R. Biswas ${ }^{4}$, S. Biswas ${ }^{4}$, J. T. Blair ${ }^{121}$, D. Blau ${ }^{83}$, C. Blume ${ }^{61}$, F. Bock ${ }^{76,96}$, A. Bogdanov ${ }^{77}$, L. Boldizsár ${ }^{140}$, M. Bombara ${ }^{40}$, M. Bonora ${ }^{35}$, J. Book ${ }^{61}$, H. Borel ${ }^{15}$, A. Borissov ${ }^{99}$, M. Borri ${ }^{128}$, E. Botta ${ }^{26}$, C. Bourjau ${ }^{84}$, P. Braun-Munzinger ${ }^{100}$, M. Bregant ${ }^{123}$, T. A. Broker ${ }^{61}$, T. A. Browning ${ }^{98}$, M. Broz ${ }^{39}$, E. J. Brucken ${ }^{46}$, E. Bruna ${ }^{113}$, G. E. Bruno ${ }^{33}$, D. Budnikov ${ }^{102}$, H. Buesching ${ }^{61}$, S. Bufalino ${ }^{26,31}$, P. Buhler ${ }^{115}$, S. A. I. Buitron ${ }^{63}$, P. Buncic ${ }^{35}$, O. Busch ${ }^{132}$, Z. Buthelezi ${ }^{67}$, J. B. Butt ${ }^{16}$, J. T. Buxton ${ }^{19}$, J. Cabala ${ }^{118}$, D. Caffarri ${ }^{35}$, H. Caines ${ }^{141}$, A. Caliva ${ }^{54}$, E. Calvo Villar ${ }^{105}$, P. Camerini ${ }^{25}$, A. A. Capon ${ }^{115}$, F. Carena ${ }^{35}$, W. Carena ${ }^{35}$, F. Carnesecchi ${ }^{12,27}$, J. Castillo Castellanos ${ }^{15}$, A. J. Castro ${ }^{129}$, E. A. R. Casula ${ }^{24,108}$, C. Ceballos Sanchez ${ }^{9}$, P. Cerello ${ }^{113}$, J. Cerkala ${ }^{118}$, B. Chang ${ }^{127}$, S. Chapeland ${ }^{35}$, M. Chartier ${ }^{128}$, J. L. Charvet ${ }^{15}$, S. Chattopadhyay ${ }^{137}$, S. Chattopadhyay ${ }^{103}$, A. Chauvin ${ }^{36,97}$, M. Cherney ${ }^{90}$, C. Cheshkov ${ }^{134}$, B. Cheynis ${ }^{134}$, V. Chibante Barroso ${ }^{35}$, D. D. Chinellato ${ }^{124}$, S. Cho ${ }^{51}$, P. Chochula ${ }^{35}$, K. Choi ${ }^{99}$, M. Chojnacki ${ }^{84}$, S. Choudhury ${ }^{137}$, P. Christakoglou ${ }^{85}$, C. H. Christensen ${ }^{84}$, P. Christiansen ${ }^{34}$, T. Chujo ${ }^{132}$, S. U. Chung ${ }^{99}$, C. Cicalo ${ }^{108}$, L. Cifarelli ${ }^{12,27}$, F. Cindolo ${ }^{107}$, J. Cleymans ${ }^{92}$, F. Colamaria ${ }^{33}$, D. Colella ${ }^{35,56}$, A. Collu ${ }^{76}$, M. Colocci ${ }^{27}$, G. Conesa Balbastre ${ }^{73}$, Z. Conesa del Valle ${ }^{52}$, M. E. Connors ${ }^{141, b}$, J. G. Contreras ${ }^{39}$, T. M. Cormier ${ }^{88}$, Y. Corrales Morales ${ }^{113}$, I. Cortés Maldonado ${ }^{2}$, P. Cortese ${ }^{32}$, M. R. Cosentino ${ }^{123,125}$, F. Costa ${ }^{35}$, J. Crkovskáa ${ }^{52}$,

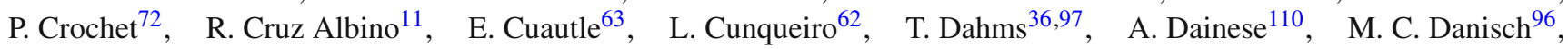
A. $\operatorname{Danu}^{59}$, D. Das ${ }^{103}$, I. Das ${ }^{103}$, S. $\operatorname{Das}^{4}$, A. $\operatorname{Dash}^{82}, \quad$ S. Dash ${ }^{48}$, S. De ${ }^{49,123}$, A. De Caro ${ }^{30}$, G. de Cataldo ${ }^{106}$, C. de Conti ${ }^{123}$, J. de Cuveland ${ }^{42}$, A. De Falco ${ }^{24}$, D. De Gruttola ${ }^{12,30}$, N. De Marco ${ }^{113}$, S. De Pasquale ${ }^{30}$, R. D. De Souza ${ }^{124}$, H. F. Degenhardt ${ }^{123}$, A. Deisting ${ }^{96,100}$, A. Deloff ${ }^{80}, \quad$ C. Deplano ${ }^{85}, \quad$ P. Dhankher ${ }^{48}, \quad$ D. Di Bari ${ }^{33}, \quad$ A. Di Mauro ${ }^{35}$,

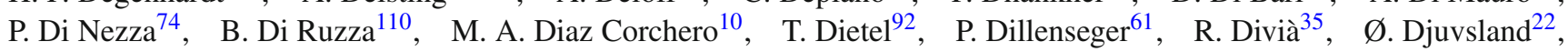
A. Dobrin ${ }^{35,59}$, D. Domenicis Gimenez ${ }^{123}$, B. Dönigus ${ }^{61}$, O. Dordic ${ }^{21}$, T. Drozhzhova ${ }^{61}$, A. K. Dubey ${ }^{137}$, A. Dubla ${ }^{100}$, L. Ducroux ${ }^{134}$, A. K. Duggal ${ }^{91}$, P. Dupieux ${ }^{72}$, R. J. Ehlers ${ }^{141}$, D. Elia ${ }^{106}$, E. Endress ${ }^{105}$, H. Engel ${ }^{60}$, E. Epple ${ }^{141}$, B. Erazmus ${ }^{116}$, F. Erhardt ${ }^{133}$, B. Espagnon ${ }^{52}$, S. Esumi ${ }^{132}$, G. Eulisse ${ }^{35}$, J. Eum ${ }^{99}$, D. Evans ${ }^{104}$, S. Evdokimov ${ }^{114}$, L. Fabbietti $^{36,97}$, D. Fabris ${ }^{110}$, J. Faivre ${ }^{73}$, A. Fantoni ${ }^{74}$, M. Fasel ${ }^{76,88}$, L. Feldkamp ${ }^{62}$, A. Feliciello ${ }^{113}$, G. Feofilov ${ }^{136}$, J. Ferencei ${ }^{87}$, A. Fernández Téllez ${ }^{2}$, E. G. Ferreiro ${ }^{17}$, A. Ferretti ${ }^{26}$, A. Festanti ${ }^{29}$, V. J. G. Feuillard ${ }^{15,72}$, J. Figiel ${ }^{120}$, M. A. S. Figueredo ${ }^{123}$, S. Filchagin ${ }^{102}$, D. Finogeev ${ }^{53}$, F. M. Fionda ${ }^{24}$, E. M. Fiore ${ }^{33}$, M. Floris ${ }^{35}$, S. Foertsch ${ }^{67}$, P. Foka ${ }^{100}$, S. Fokin ${ }^{83}$, E. Fragiacomo ${ }^{112}$, A. Francescon ${ }^{35}$, A. Francisco ${ }^{116}$, U. Frankenfeld ${ }^{100}$, G. G. Fronze ${ }^{26}$, U. Fuchs $^{35}$, C. Furget ${ }^{73}$, A. Furs ${ }^{53}$, M. Fusco Girard ${ }^{30}$, J. J. Gaardhøje ${ }^{84}$, M. Gagliardi ${ }^{26}$, A. M. Gago ${ }^{105}$, K. Gajdosova ${ }^{84}$, M. Gallio ${ }^{26}$, C. D. Galvan ${ }^{122}$, D. R. Gangadharan ${ }^{76}$, P. Ganoti ${ }^{79}$, C. $\mathrm{Gao}^{7}$, C. Garabatos ${ }^{100}$, E. Garcia-Solis ${ }^{13}$, K. Garg ${ }^{28}$, P. Garg ${ }^{49}$, C. Gargiulo ${ }^{35}$, P. Gasik ${ }^{36,97}$, E. F. Gauger ${ }^{121}$, M. B. Gay Ducati ${ }^{64}$, M. Germain ${ }^{116}$, P. Ghosh ${ }^{137}$, S. K. Ghosh ${ }^{4}$, P. Gianotti ${ }^{74}$, P. Giubellino ${ }^{35,113}$, P. Giubilato ${ }^{29}$, E. Gladysz-Dziadus ${ }^{120}$, P. Glässel ${ }^{96}$, D. M. Goméz Coral ${ }^{65}$, A. Gomez Ramirez $z^{60}$, A. S. Gonzalez ${ }^{35}$, V. Gonzalez ${ }^{10}$, P. González-Zamora ${ }^{10}$, S. Gorbunov ${ }^{42}$, L. Görlich ${ }^{120}$, S. Gotovac ${ }^{119}$, V. Grabski ${ }^{65}$, L. K. Graczykowski ${ }^{138}$, K. L. Graham ${ }^{104}$, L. Greiner ${ }^{76}$, A. Grelli ${ }^{54}$, C. Grigoras ${ }^{35}$, V. Grigoriev ${ }^{77}$, A. Grigoryan ${ }^{1}$, S. Grigoryan ${ }^{68}$, N. Grion ${ }^{112}$, J. M. Gronefeld ${ }^{100}$, F. Grosa ${ }^{31}$, J. F. Grosse-Oetringhaus ${ }^{35}$, R. Grosso ${ }^{100}$, L. Gruber ${ }^{115}$, F. R. Grull ${ }^{60}$, F. Guber ${ }^{53}$, R. Guernane ${ }^{35,73}$, B. Guerzoni ${ }^{27}$, K. Gulbrandsen ${ }^{84}$, T. Gunji ${ }^{131}$, A. Gupta ${ }^{93}$, R. Gupta ${ }^{93}$, I. B. Guzman ${ }^{2}$, R. Haake ${ }^{35,62}$, C. Hadjidakis ${ }^{52}$, H. Hamagaki ${ }^{78,131}$, G. Hamar ${ }^{140}$, J. C. Hamon ${ }^{66}$, J. W. Harris ${ }^{141}$, A. Harton ${ }^{13}$, D. Hatzifotiadou ${ }^{107}$, S. Hayashi1 ${ }^{131}$, S. T. Heckel ${ }^{61}$, E. Hellbär ${ }^{61}$, H. Helstrup 37 A. Herghelegiu ${ }^{81}$, G. Herrera Corral ${ }^{11}$, F. Herrmann ${ }^{62}$, B. A. Hess ${ }^{95}$, K. F. Hetland ${ }^{37}$, H. Hillemanns ${ }^{35}$, B. Hippolyte ${ }^{66}$, J. Hladky ${ }^{57}$, D. Horak ${ }^{39}$ ， R. Hosokawa ${ }^{132}$, P. Hristov ${ }^{35}$, C. Hughes ${ }^{129}$, T. J. Humanic ${ }^{19}$, N. Hussain ${ }^{44}$, T. Hussain ${ }^{18}$, D. Hutter ${ }^{42}$, D. S. Hwang ${ }^{20}$, R. Ilkaev ${ }^{102}$, M. Inaba ${ }^{132}$, M. Ippolitov ${ }^{77,83}$, M. Irfan ${ }^{18}$, V. Isakov ${ }^{53}$ M. S. Islam ${ }^{49}$, M. Ivanov ${ }^{35,100}$, 
V. Ivanov ${ }^{89}$ ，V. Izucheev ${ }^{114}$ ， B. Jacak ${ }^{76}$ ，N. Jacazio ${ }^{27}$ ，P. M. Jacobs ${ }^{76}$ ，M. B. Jadhav ${ }^{48}$ ，S. Jadlovska ${ }^{118}$, J. Jadlovsky ${ }^{118}$, C. Jahnke ${ }^{36}$, M. J. Jakubowska ${ }^{138}$ ， M. A. Janik ${ }^{138}$ ， P. H. S. Y. Jayarathna ${ }^{126}$, C. Jena ${ }^{82}$ ， S. Jena ${ }^{126}, \quad$ M. Jercic ${ }^{133}$, R. T. Jimenez Bustamante ${ }^{100}$ ， P. G. Jones ${ }^{104}$, A. Jusko ${ }^{104}$ ， P. Kalinak ${ }^{56}$ ， A. Kalweit ${ }^{35}$ ， J. H. Kang ${ }^{142}$, V. Kaplin ${ }^{77}$, S. Kar ${ }^{137}$, A. Karasu Uysal ${ }^{71}$, O. Karavichev ${ }^{53}$, T. Karavicheva ${ }^{53}$, L. Karayan ${ }^{96,100}$, E. Karpechev ${ }^{53}$, U. Kebschull ${ }^{60}$, R. Keidel ${ }^{143}$, D. L. D. Keijdener ${ }^{54}$, M. Keil ${ }^{35}$, M. Mohisin Khan ${ }^{18, c}$, P. Khan ${ }^{103}$ ， S. A. Khan ${ }^{137}$, A. Khanzadeev ${ }^{89}$, Y. Kharlov ${ }^{114}$, A. Khatun ${ }^{18}$, A. Khuntia ${ }^{49}$, M. M. Kielbowicz ${ }^{120}$, B. Kileng ${ }^{37}$, D. W. Kim${ }^{43}$, D. J. Kim ${ }^{127}$, D. Kim ${ }^{142}$,

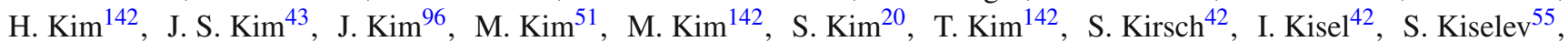
A. Kisiel ${ }^{138}$, G. Kiss ${ }^{140}$, J. L. Klay ${ }^{6}$, C. Klein ${ }^{61}$, J. Klein ${ }^{35}$, C. Klein-Bösing ${ }^{62}$, S. Klewin ${ }^{96}$, A. Kluge ${ }^{35}$, M. L. Knichel ${ }^{96}$, A. G. Knospe ${ }^{126}$, C. Kobdaj ${ }^{117}$, M. Kofarago ${ }^{35}$, T. Kollegger ${ }^{100}$, A. Kolojvari ${ }^{136}$, V. Kondratiev ${ }^{136}$, N. Kondratyeva ${ }^{77}$, E. Kondratyuk ${ }^{114}$, A. Konevskikh ${ }^{53}$, M. Kopcik ${ }^{118}$, M. Kour ${ }^{93}$, C. Kouzinopoulos ${ }^{35}$, O. Kovalenko ${ }^{80}$, V. Kovalenko ${ }^{136}$, M. Kowalski ${ }^{120}$, G. Koyithatta Meethaleveedu ${ }^{48}$, I. Králik ${ }^{56}$, A. Kravčáková ${ }^{40}$, M. Krivda ${ }^{56,104}$, F. Krizek ${ }^{87}$, E. Kryshen ${ }^{89}$, M. Krzewicki ${ }^{42}$, A. M. Kubera ${ }^{19}$, V. Kučera ${ }^{87}$, C. Kuhn ${ }^{66}$, P. G. Kuijer ${ }^{85}$, A. $\operatorname{Kumar}^{93}$, J. Kumar ${ }^{48}$, L. Kumar ${ }^{91}$, S. Kumar ${ }^{48}$, S. Kundu ${ }^{82}$, P. Kurashvili ${ }^{80}$, A. Kurepin ${ }^{53}$, A. B. Kurepin' ${ }^{53}$, A. Kuryakin ${ }^{102}$, S. Kushpil ${ }^{87}$, M. J. Kweon ${ }^{51}$, Y. Kwon ${ }^{142}$,

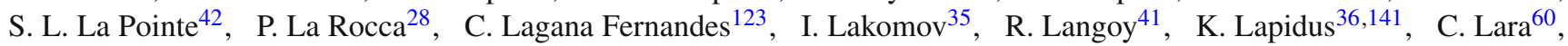
A. Lardeux ${ }^{15,21}$, A. Lattuca ${ }^{26}$, E. Laudi ${ }^{35}$, R. Lavicka ${ }^{39}$, L. Lazaridis ${ }^{35}$, R. Lea ${ }^{25}$, L. Leardini96, S. Lee ${ }^{142}$, F. Lehas ${ }^{85}$, S. Lehner ${ }^{15}$, J. Lehrbach ${ }^{42}$, R. C. Lemmon ${ }^{86}$, V. Lenti ${ }^{106}$, E. Leogrande ${ }^{54}$, I. León Monzón ${ }^{122}$, P. Lévai ${ }^{140}, \mathrm{~S} \mathrm{Li}^{7}, \mathrm{X} \mathrm{Li}^{14}$, J. Lien ${ }^{41}$, R. Lietava ${ }^{104}$, S. Lindal ${ }^{21}$, V. Lindenstruth ${ }^{42}$, C. Lippmann ${ }^{100}$, M. A. Lisa ${ }^{19}$, V. Litichevskyi ${ }^{46}$, H. M. Ljunggren ${ }^{34}$, W. J. Llope ${ }^{139}$, D. F. Lodato ${ }^{54}$, P. I. Loenne ${ }^{22}$, V. Loginov ${ }^{77}$, C. Loizides ${ }^{76}$, P. Loncar ${ }^{119}$, X. Lopez ${ }^{72}$, E. López Torres ${ }^{9}$, A. Lowe ${ }^{140}$, P. Luettig ${ }^{61}$, M. Lunardon ${ }^{29}$, G. Luparello ${ }^{25}$, M. Lupi ${ }^{35}$, T. H. Lutz ${ }^{141}$, A. Maevskaya ${ }^{53}$, M. Mager ${ }^{35}$, S. Mahajan ${ }^{93}$, S. M. Mahmood ${ }^{21}$, A. Maire ${ }^{66}$, R. D. Majka ${ }^{141}$, M. Malaev ${ }^{89}$, I. Maldonado Cervantes ${ }^{63}$, L. Malinina ${ }^{68, d}$, D. Mal'Kevich ${ }^{55}$, P. Malzacher ${ }^{100}$, A. Mamonov ${ }^{102}$, V. Manko ${ }^{83}$, F. Manso ${ }^{72}$, V. Manzari ${ }^{106}$, Y. Mao ${ }^{7}$, M. Marchisone ${ }^{67,130}$, J. Mareš ${ }^{57}$, G. V. Margagliotti ${ }^{25}$, A. Margotti ${ }^{107}$, J. Margutti ${ }^{54}$, A. Marín ${ }^{100}$, C. Markert ${ }^{121}$, M. Marquard ${ }^{61}$, N. A. Martin ${ }^{100}$, P. Martinengo $^{35}$, M. I. Martínez ${ }^{2}$ ， G. Martínez García ${ }^{116}$, M. Martinez Pedreira ${ }^{35}$, A. Mas ${ }^{123}$, S. Masciocchi ${ }^{100}$, M. Masera ${ }^{26}$, A. Masoni ${ }^{108}$, A. Mastroserio ${ }^{33}$, A. M. Mathis ${ }^{36,97}$ A. Matyja ${ }^{120,129}$, C. Mayer ${ }^{120}$, J. Mazer ${ }^{129}$, M. Mazzilli ${ }^{33}$, M. A. Mazzoni ${ }^{111}$, F. Meddi' ${ }^{23}$, Y. Melikyann ${ }^{77}$, A. Menchaca-Rocha ${ }^{65}$, E. Meninno ${ }^{30}$, J. Mercado Pérez ${ }^{96}$, M. Meres ${ }^{38}$, S. Mhlanga ${ }^{92}$, Y. Miake ${ }^{132}$, M. M. Mieskolainen ${ }^{46}$, K. Mikhaylov ${ }^{55,68}$, L. Milano ${ }^{76}$, J. Milosevic ${ }^{21}$, A. Mischke ${ }^{54}$, A. N. Mishra ${ }^{49}$, T. Mishra ${ }^{58}$, D. Miśkowiec ${ }^{100}$, J. Mitra ${ }^{137}$, C. M. Mitu ${ }^{59}$, N. Mohammadi ${ }^{54}$, B. Mohanty ${ }^{82}$, L. Molnar ${ }^{16}$, E. Montes $^{10}$, D. A. Moreira De Godoy ${ }^{62}$, L. A. P. Moreno ${ }^{2}$, S. Moretto ${ }^{29}$, A. Morreale ${ }^{116}$, A. Morsch ${ }^{35}$, V. Muccifora ${ }^{74}$, E. Mudnic ${ }^{119}$, D. Mühlheim ${ }^{62}$, S. Muhuri ${ }^{137}$, M. Mukherjee ${ }^{137}$, J. D. Mulligan ${ }^{141}$, M. G. Munhoz ${ }^{123}$, K. Münning ${ }^{45}$, R. H. Munzer ${ }^{36,61,97}$, H. Murakami ${ }^{131}$, S. Murray ${ }^{67}, \quad$ L. Musa ${ }^{35}$, J. Musinsky ${ }^{56}$, C. J. Myers ${ }^{126}$, B. $\mathrm{Naik}^{48}$, R. Nair ${ }^{80}$, B. K. Nandi ${ }^{48}$, R. Nania ${ }^{107}$, E. Nappi ${ }^{106}$, M. U. Naru ${ }^{16}$, H. Natal da Luz ${ }^{123}$, C. Nattrass ${ }^{129}$, S. R. Navarro ${ }^{2}$, K. Nayak ${ }^{82}$, R. Nayak ${ }^{48}$, T. K. Nayak ${ }^{137}$, S. Nazarenko ${ }^{102}$, A. Nedosekin ${ }^{55}$, R. A. Negrao De Oliveira ${ }^{35}$, L. Nellen ${ }^{63}$, S. V. Nesbo ${ }^{37}$, F. Ng ${ }^{126}$, M. Nicassio ${ }^{100}$, M. Niculescu ${ }^{59}$, J. Niedziela ${ }^{35}$, B. S. Nielsen ${ }^{84}$, S. Nikolaev ${ }^{83}$, S. Nikulin ${ }^{83}$, V. Nikulin ${ }^{89}$, F. Noferini ${ }^{12,107}$, P. Nomokonov ${ }^{68}$, G. Nooren ${ }^{54}$, J. C. C. Noris ${ }^{2}$, J. Norman ${ }^{128}$, A. Nyanin ${ }^{83}$, J. Nystrand ${ }^{22}$, H. Oeschler ${ }^{96}$, S. Oh ${ }^{141}$, A. Ohlson ${ }^{35,96}$, T. Okubo ${ }^{47}$, L. Olah ${ }^{140}$, J. Oleniacz ${ }^{138}$, A. C. Oliveira Da Silva ${ }^{123}$, M. H. Oliver ${ }^{141}$, J. Onderwaater ${ }^{100}$, C. Oppedisano ${ }^{113}$, R. Orava ${ }^{46}$, M. Oravec ${ }^{118}$, A. Ortiz Velasquez ${ }^{63}$, A. Oskarsson ${ }^{34}$, J. Otwinowski ${ }^{120}$, K. Oyama ${ }^{78}$, M. Ozdemir ${ }^{61}$, Y. Pachmayer ${ }^{96}$, V. Pacik ${ }^{84}$, D. Pagano ${ }^{26,135}$, P. Pagano ${ }^{30}$, G. Paić 63 , S. K. Pal ${ }^{137}$, P. Palni ${ }^{7}$, J. Pan ${ }^{139}$, A. K. Pandey ${ }^{48}$, S. Panebianco ${ }^{15}$, V. Papikyan ${ }^{1}$, G. S. Pappalardo ${ }^{109}$, P. Pareek ${ }^{49}$, J. Park ${ }^{51}$, W. J. Park ${ }^{100}$, S. Parmar91, A. Passfeld ${ }^{62}$, V. Paticchio ${ }^{106}$, R. N. Patra ${ }^{137}$, B. Paul ${ }^{113}$, H. Pei $^{7}$, T. Peitzmann ${ }^{54}$, X. Peng ${ }^{7}$, L. G. Pereira ${ }^{64}$, H. Pereira Da Costa ${ }^{15}$, D. Peresunko ${ }^{77,83}$, E. Perez Lezama ${ }^{61}$, V. Peskov ${ }^{61}$, Y. Pestov ${ }^{5}$, V. Petráček ${ }^{39}$, V. Petrov ${ }^{114}$, M. Petrovici ${ }^{81}$, C. Petta ${ }^{28}$, R. P. Pezzi $^{64}$, S. Piano ${ }^{112}$, M. Pikna ${ }^{38}$, P. Pillot ${ }^{116}$, L. O. D. L. Pimentel ${ }^{84}$, O. Pinazza $^{35,107}$, L. Pinsky ${ }^{126}$, D. B. Piyarathna ${ }^{126}$, M. Pł oskoń ${ }^{76}, \quad$ M. Planinic ${ }^{133}$, J. Pluta ${ }^{138}$, S. Pochybova ${ }^{140}$, P. L. M. Podesta-Lerma ${ }^{122}$, M. G. Poghosyan ${ }^{88}$, B. Polichtchouk ${ }^{114}$, N. Poljak ${ }^{133}$, W. Poonsawat ${ }^{117}$, A. Pop ${ }^{81}$, H. Poppenborg ${ }^{62}$, S. Porteboeuf-Houssais ${ }^{72}$, J. Porter ${ }^{76}$, J. Pospisil ${ }^{87}$, V. Pozdniakov ${ }^{68}$, S. K. Prasad ${ }^{4}$, R. Preghenella ${ }^{35,107}$, F. Prino ${ }^{113}$, C. A. Pruneau ${ }^{139}$, I. Pshenichnov ${ }^{53}$, M. Puccio ${ }^{26}$, G. Puddu ${ }^{24}$, P. Pujahari ${ }^{139}$, V. Punin ${ }^{102}$, J. Putschke ${ }^{139}$, H. Qvigstad ${ }^{21}$, A. Rachevski ${ }^{112}$, S. Raha ${ }^{4}$, S. Rajput ${ }^{93}$, J. Rak ${ }^{127}$, A. Rakotozafindrabe ${ }^{15}$, L. Ramello ${ }^{32}$, F. Rami ${ }^{66}$, D. B. Rana ${ }^{126}$, R. Raniwala ${ }^{94}$, S. Raniwala ${ }^{94}$, S. S. Räsänen ${ }^{46}$, B. T. Rascanu ${ }^{61}$, D. Rathee ${ }^{91}$, V. Ratza ${ }^{45}$, I. Ravasenga ${ }^{31}$, K. F. Read ${ }^{88,129}$, K. Redlich ${ }^{80}$, A. Rehman ${ }^{22}$, P. Reichelt ${ }^{61}$, F. Reidt ${ }^{35}$, X. Ren ${ }^{7}$, R. Renfordt ${ }^{61}$, A. R. Reolon ${ }^{74}$, A. Reshetin ${ }^{53}$, K. Reygers ${ }^{96}$, V. Riabov ${ }^{89}$, R. A. Ricci $^{75}$, T. Richert ${ }^{34,54}$, M. Richter ${ }^{21}$, P. Riedler ${ }^{35}$, W. Riegler ${ }^{35}$, F. Riggi ${ }^{28}$, C. Ristea ${ }^{59}$, M. Rodríguez Cahuantzi ${ }^{2}$, K. Røed ${ }^{21}$, E. Rogochaya ${ }^{68}$, D. Rohr ${ }^{42}$, D. Röhrich ${ }^{22}$, F. Ronchetti ${ }^{35,74}$, L. Ronflette ${ }^{116}$, P. Rosnet ${ }^{72}$, A. Rossi ${ }^{29}$, F. Roukoutakis ${ }^{79}$, A. Roy ${ }^{49}$, C. Roy ${ }^{66}$, P. Roy ${ }^{103}$, A. J. Rubio Montero ${ }^{10}$, R. Rui ${ }^{25}$ ， R. Russo ${ }^{26}$, E. Ryabinkin ${ }^{83}$, Y. Ryabov ${ }^{89}$ ，A. Rybicki ${ }^{120}$ ， S. Saarinen ${ }^{46}$ ， S. Sadhu ${ }^{137}$, S. Sadovsky ${ }^{114}$, K. Šafařík ${ }^{35}$, B. Sahlmuller ${ }^{61}$, B. Sahoo ${ }^{48}$, P. Sahoo ${ }^{49}$, R. Sahoo ${ }^{49}$, S. Sahoo ${ }^{58}$, P. K. Sahu ${ }^{58}$, J. Saini ${ }^{137}$, S. Sakai ${ }^{74,132}$, 
M. A. Saleh ${ }^{139}$, J. Salzwedel ${ }^{19}$, S. Sambyal ${ }^{93}$, V. Samsonov ${ }^{77,89}$, A. Sandoval ${ }^{65}$, D. Sarkar ${ }^{137}$, N. Sarkar ${ }^{137}$, P. Sarma ${ }^{44}$, M. H. P. Sas ${ }^{54}$ ， E. Scapparone ${ }^{107}$ ，F. Scarlassara ${ }^{29}$ ，R. P. Scharenberg ${ }^{98 ， C . ~ S c h i a u a ~}{ }^{81 ， R . ~ S c h i c k e r}{ }^{96}$ ，C. Schmidt ${ }^{100}$, H. R. Schmidt ${ }^{95}$, M. O. Schmidt ${ }^{96}$, M. Schmidt ${ }^{95}$, J. Schukraft ${ }^{35}$, Y. Schutz ${ }^{35,66,116}$, K. Schwarz ${ }^{100}$, K. Schweda ${ }^{100}$, G. Scioli ${ }^{27}$, E. Scomparin ${ }^{113}$, R. Scott ${ }^{129}$, M. Šefčík ${ }^{40}$, J. E. Seger ${ }^{90}$, Y. Sekiguchi ${ }^{131}$, D. Sekihata ${ }^{47}$, I. Selyuzhenkov ${ }^{100}$, K. Senosi ${ }^{67}$, S. Senyukov ${ }^{3,35,66}$, E. Serradilla ${ }^{10,65}$, P. Sett ${ }^{48}$, A. Sevcenco ${ }^{59}$, A. Shabanov ${ }^{53}$, A. Shabetai ${ }^{116}$, O. Shadura ${ }^{3}$, R. Shahoyan ${ }^{35}$, A. Shangaraev ${ }^{114}$, A. Sharma ${ }^{93}$, A. Sharma ${ }^{91}$, M. Sharma ${ }^{93}$, M. Sharma ${ }^{93}$, N. Sharma ${ }^{91,129}$, A. I. Sheikh ${ }^{137}$, K. Shigaki ${ }^{47}$, Q. Shou ${ }^{7}$, K. Shtejer ${ }^{9,26}$, Y. Sibiriak ${ }^{83}$, S. Siddhanta ${ }^{108}$, K. M. Sielewicz ${ }^{35}$, T. Siemiarczuk ${ }^{80}$, D. Silvermyr ${ }^{34}$, C. Silvestre ${ }^{73}$, G. Simatovic ${ }^{133}$ ， G. Simonetti ${ }^{35}$ ， R. Singaraju ${ }^{137}$ ， R. $\operatorname{Singh}^{82}$, V. Singhal ${ }^{137}$, T. Sinha ${ }^{103}$, B. $\operatorname{Sitar}^{38}$, M. Sitta ${ }^{32}$, T. B. Skaali ${ }^{21}$, M. Slupecki ${ }^{127}$, N. Smirnov ${ }^{141}$, R. J. M. Snellings ${ }^{54}$, T. W. Snellman ${ }^{127}$, J. Song ${ }^{99}$, M. Song ${ }^{142}$, F. Soramel ${ }^{29}$ ， S. Sorensen ${ }^{129}$ ， F. Sozzi ${ }^{100}$, E. Spiriti ${ }^{74}$, I. Sputowska ${ }^{120}$ ， B. K. Srivastava ${ }^{98}$ ， J. Stachel ${ }^{96}$, I. Stan ${ }^{59}$, P. Stankus ${ }^{88}$, E. Stenlund ${ }^{34}$, J. H. Stiller ${ }^{96}$, D. Stocco ${ }^{116}$, P. Strmen ${ }^{38}$, A. A. P. Suaide ${ }^{123}$, T. Sugitate ${ }^{47}$, C. Suire ${ }^{52}$, M. Suleymanov ${ }^{16}$, M. Suljic ${ }^{25}$, R. Sultanov ${ }^{55}$, M. Šumbera ${ }^{87}$, S. Sumowidagdo ${ }^{50}$, K. Suzuki ${ }^{115}$, S. Swain ${ }^{58}$, A. Szabo ${ }^{38}$, I. Szarka ${ }^{38}$, A. Szczepankiewicz ${ }^{138}$, M. Szymanski ${ }^{138}$, U. Tabassam ${ }^{16}$, J. Takahashi ${ }^{124}$, G. J. Tambave ${ }^{22}$, N. Tanaka ${ }^{132}$, M. Tarhini ${ }^{52}$, M. Tariq ${ }^{18}$, M. G. Tarzila ${ }^{81}$, A. Tauro ${ }^{35}$, G. Tejeda Muñoz ${ }^{2}$, A. Telesca ${ }^{35}$, K. Terasaki ${ }^{131}$, C. Terrevoli ${ }^{29}$, B. Teyssier ${ }^{134}$, D. Thakur ${ }^{49}$, D. Thomas ${ }^{121}$, R. Tieulent ${ }^{134}$, A. Tikhonov ${ }^{53}$, A. R. Timmins ${ }^{126}$, A. Toia ${ }^{61}$, S. Tripathy ${ }^{49}$, S. Trogolo ${ }^{26}$, G. Trombetta ${ }^{33}$, V. Trubnikov ${ }^{3}$, W. H. Trzaska ${ }^{127}$, B. A. Trzeciak ${ }^{54}$, T. Tsuji ${ }^{131}$, A. Tumkin ${ }^{102}$, R. Turrisi ${ }^{110}$, T. S. Tveter ${ }^{21}$, K. Ullaland ${ }^{22}$, E. N. Umaka ${ }^{126}$, A. Uras ${ }^{134}$, G. L. Usai ${ }^{24}$, A. Utrobicic ${ }^{133}$, M. Vala ${ }^{56,118}$, J. Van Der Maarel ${ }^{54}$, J. W. Van Hoorne ${ }^{35}$, M. van Leeuwen ${ }^{54}$, T. Vanat ${ }^{87}$, P. Vande Vyvre ${ }^{35}$, D. Varga ${ }^{140}$, A. Vargas ${ }^{2}$, M. Vargyas ${ }^{127}$, R. Varma ${ }^{48}$, M. Vasileiou ${ }^{79}$, A. Vasiliev ${ }^{83}$, A. Vauthier ${ }^{73}$, O. Vázquez Doce ${ }^{36,97}$, V. Vechernin ${ }^{136}$, A. M. Veen ${ }^{54}$, A. Velure ${ }^{22}$, E. Vercellin ${ }^{26}$, S. Vergara Limón ${ }^{2}$, R. Vernet ${ }^{8}$, R. Vértesi ${ }^{140}$, L. Vickovic ${ }^{119}$, S. Vigolo ${ }^{54}$, J. Viinikainen ${ }^{127}$, Z. Vilakazi ${ }^{130}$, $\begin{array}{lll}\text { O. Villalobos Baillie } & 104 & \text { A. Villatoro Tello } \\ & \end{array}$ A. Vinogradov ${ }^{83}, \quad$ L. Vinogradov ${ }^{136}, \quad$ T. Virgili ${ }^{30}, \quad$ V. Vislavicius ${ }^{34}$, A. Vodopyanov ${ }^{68}$, M. A. Völk1 ${ }^{96}$, K. Voloshin55, S. A. Voloshin ${ }^{139}$, G. Volpe ${ }^{33}$, B. von Haller ${ }^{35}$, I. Vorobyev ${ }^{36,97}$, D. Voscek ${ }^{118}$, D. Vranic ${ }^{35,100}$, J. Vrláková4 ${ }^{40}$, B. Wagner ${ }^{22}$, J. Wagner ${ }^{100}$, H. Wang ${ }^{54}$, M. Wang ${ }^{7}$, D. Watanabe ${ }^{132}$, Y. Watanabe ${ }^{131}$, M. Weber ${ }^{115}$, S. G. Weber ${ }^{100}$, D. F. Weiser ${ }^{96}$, J. P. Wessels ${ }^{62}$, U. Westerhoff ${ }^{62}$, A. M. Whitehead ${ }^{92}$, J. Wiechula ${ }^{61}$, J. Wikne ${ }^{21}$, G. Wilk ${ }^{80}$, J. Wilkinson ${ }^{96}$, G. A. Willems ${ }^{62}$, M. C. S. Williams ${ }^{107}$, B. Windelband ${ }^{96}$, W. E. Witt ${ }^{129}$, S. Yalcin ${ }^{71}$, P. Yang ${ }^{7}$, S. Yano ${ }^{47}$, Z. Yin ${ }^{7}$, H. Yokoyama ${ }^{73,132}$, I.-K. Yoo ${ }^{35,99}$, J. H. Yoon ${ }^{51}$, V. Yurchenko ${ }^{3}$, V. Zaccolo ${ }^{84,113}$, A. Zaman ${ }^{16}$, C. Zampolli ${ }^{35}$, H. J. C. Zanoli ${ }^{123}$, S. Zaporozhets ${ }^{68}$, N. Zardoshti ${ }^{104}$, A. Zarochentsev ${ }^{136}$, P. Závada ${ }^{57}$, N. Zaviyalov ${ }^{102}$, H. Zbroszczyk ${ }^{138}$, M. Zhalov ${ }^{89}$, H. Zhang ${ }^{7,22}$, X. Zhang ${ }^{7,76}$, Y. Zhang ${ }^{7}$, C. Zhang ${ }^{54}$, Z. Zhang ${ }^{7}$, C. Zhao ${ }^{21}$, N. Zhigareva ${ }^{55}$, D. Zhou ${ }^{7}$, Y. Zhou ${ }^{84}$, Z. Zhou ${ }^{22}$, H. Zhu ${ }^{22,7}$, J. Zhu ${ }^{7,116}$, X. Zhu ${ }^{7}$, A. Zichichi ${ }^{12,27}$, A. Zimmermann ${ }^{96}$, M. B. Zimmermann ${ }^{35,62}$, S. Zimmermann ${ }^{115}$, G. Zinovjev ${ }^{3}$, J. Zmeskal ${ }^{115}$

${ }^{1}$ A.I. Alikhanyan National Science Laboratory (Yerevan Physics Institute) Foundation, Yerevan, Armenia

2 Benemérita Universidad Autónoma de Puebla, Puebla, Mexico

${ }^{3}$ Bogolyubov Institute for Theoretical Physics, Kiev, Ukraine

${ }^{4}$ Department of Physics and Centre for Astroparticle Physics and Space Science (CAPSS), Bose Institute, Kolkata, India

${ }^{5}$ Budker Institute for Nuclear Physics, Novosibirsk, Russia

${ }^{6}$ California Polytechnic State University, San Luis Obispo, United States

${ }^{7}$ Central China Normal University, Wuhan, China

${ }^{8}$ Centre de Calcul de l'IN2P3, Villeurbanne, Lyon, France

${ }^{9}$ Centro de Aplicaciones Tecnológicas y Desarrollo Nuclear (CEADEN), Havana, Cuba

${ }^{10}$ Centro de Investigaciones Energéticas Medioambientales y Tecnológicas (CIEMAT), Madrid, Spain

${ }^{11}$ Centro de Investigación y de Estudios Avanzados (CINVESTAV), Mexico City, Mérida, Mexico

12 Centro Fermi-Museo Storico della Fisica e Centro Studi e Ricerche "Enrico Fermi', Rome, Italy

${ }^{13}$ Chicago State University, Chicago, United States

${ }^{14}$ China Institute of Atomic Energy, Beijing, China

${ }^{15}$ Commissariat à l'Energie Atomique, IRFU, Saclay, France

${ }^{16}$ COMSATS Institute of Information Technology (CIIT), Islamabad, Pakistan

${ }^{17}$ Departamento de Física de Partículas and IGFAE, Universidad de Santiago de Compostela, Santiago de Compostela, Spain

${ }^{18}$ Department of Physics, Aligarh Muslim University, Aligarh, India

${ }^{19}$ Department of Physics, Ohio State University, Columbus, Ohio, United States

${ }^{20}$ Department of Physics, Sejong University, Seoul, South Korea

${ }^{21}$ Department of Physics, University of Oslo, Oslo, Norway 
22 Department of Physics and Technology, University of Bergen, Bergen, Norway

${ }^{23}$ Dipartimento di Fisica dell'Università 'La Sapienza' and Sezione INFN, Rome, Italy

${ }^{24}$ Dipartimento di Fisica dell'Università and Sezione INFN, Cagliari, Italy

${ }^{25}$ Dipartimento di Fisica dell'Università and Sezione INFN, Trieste, Italy

${ }^{26}$ Dipartimento di Fisica dell'Università and Sezione INFN, Turin, Italy

${ }^{27}$ Dipartimento di Fisica e Astronomia dell'Università and Sezione INFN, Bologna, Italy

${ }^{28}$ Dipartimento di Fisica e Astronomia dell'Università and Sezione INFN, Catania, Italy

${ }^{29}$ Dipartimento di Fisica e Astronomia dell'Università and Sezione INFN, Padovaa, Italy

${ }^{30}$ Dipartimento di Fisica ‘E.R. Caianiello' dell’Università and Gruppo Collegato INFN, Salerno, Italy

${ }^{31}$ Dipartimento DISAT del Politecnico and Sezione INFN, Turin, Italy

32 Dipartimento di Scienze e Innovazione Tecnologica dell'Università del Piemonte Orientale and INFN Sezione di Torino, Alessandria, Italy

${ }^{33}$ Dipartimento Interateneo di Fisica 'M. Merlin' and Sezione INFN, Bari, Italy

${ }^{34}$ Division of Experimental High Energy Physics, University of Lund, Lund, Sweden

${ }^{35}$ European Organization for Nuclear Research (CERN), Geneva, Switzerland

${ }^{36}$ Excellence Cluster Universe, Technische Universität München, Munich, Germany

${ }^{37}$ Faculty of Engineering, Bergen University College, Bergen, Norway

${ }^{38}$ Faculty of Mathematics, Physics and Informatics, Comenius University, Bratislava, Slovakia

${ }^{39}$ Faculty of Nuclear Sciences and Physical Engineering, Czech Technical University in Prague, Prague, Czech Republic

${ }^{40}$ Faculty of Science, P.J. Šafárik University, Kosice, Slovakia

${ }^{41}$ Faculty of Technology, Buskerud and Vestfold University College, Tonsberg, Norway

${ }^{42}$ Frankfurt Institute for Advanced Studies, Johann Wolfgang Goethe-Universität Frankfurt, Frankfurt, Germany

${ }^{43}$ Gangneung-Wonju National University, Gangneung, South Korea

${ }^{44}$ Department of Physics, Gauhati University, Guwahati, India

${ }^{45}$ Helmholtz-Institut für Strahlen- und Kernphysik, Rheinische Friedrich-Wilhelms-Universität Bonn, Bonn, Germany

${ }^{46}$ Helsinki Institute of Physics (HIP), Helsinki, Finland

${ }^{47}$ Hiroshima University, Hiroshima, Japan

${ }^{48}$ Indian Institute of Technology Bombay (IIT), Mumbai, India

${ }^{49}$ Indian Institute of Technology Indore, Indore, India

${ }^{50}$ Indonesian Institute of Sciences, Jakarta, Indonesia

${ }^{51}$ Inha University, Incheon, South Korea

52 Institut de Physique Nucléaire d'Orsay (IPNO), Université Paris-Sud, CNRS-IN2P3, Orsay, France

${ }^{53}$ Institute for Nuclear Research, Academy of Sciences, Moscow, Russia

${ }^{54}$ Institute for Subatomic Physics of Utrecht University, Utrecht, Netherlands

55 Institute for Theoretical and Experimental Physics, Moscow, Russia

${ }^{56}$ Institute of Experimental Physics, Slovak Academy of Sciences, Košice, Slovakia

${ }^{57}$ Institute of Physics, Academy of Sciences of the Czech Republic, Prague, Czech Republic

${ }^{58}$ Institute of Physics, Bhubaneswar, India

${ }^{59}$ Institute of Space Science (ISS), Bucharest, Romania

${ }^{60}$ Institut für Informatik, Johann Wolfgang Goethe-Universität Frankfurt, Frankfurt, Germany

${ }^{61}$ Institut für Kernphysik, Johann Wolfgang Goethe-Universität Frankfurt, Frankfurt, Germany

${ }^{62}$ Institut für Kernphysik, Westfälische Wilhelms-Universität Münster, Münster, Germany

${ }^{63}$ Instituto de Ciencias Nucleares, Universidad Nacional Autónoma de México, Mexico City, Mexico

${ }^{64}$ Instituto de Física, Universidade Federal do Rio Grande do Sul (UFRGS), Porto Alegre, Brazil

${ }^{65}$ Instituto de Física, Universidad Nacional Autónoma de México, Mexico City, Mexico

${ }^{66}$ Institut Pluridisciplinaire Hubert Curien (IPHC), Université de Strasbourg, CNRS-IN2P3, Strasbourg, France

67 iThemba LABS, National Research Foundation, Somerset West, South Africa

${ }^{68}$ Joint Institute for Nuclear Research (JINR), Dubna, Russia

${ }^{69}$ Konkuk University, Seoul, South Korea

${ }^{70}$ Korea Institute of Science and Technology Information, Daejeon, South Korea

${ }^{71}$ KTO Karatay University, Konya, Turkey

${ }^{72}$ Laboratoire de Physique Corpusculaire (LPC), Clermont Université, Université Blaise Pascal, CNRS-IN2P3, Clermont-Ferrand, France 
${ }^{73}$ Laboratoire de Physique Subatomique et de Cosmologie, Université Grenoble-Alpes, CNRS-IN2P3, Grenoble, France

${ }^{74}$ Laboratori Nazionali di Frascati, INFN, Frascati, Italy

${ }^{75}$ Laboratori Nazionali di Legnaro, INFN, Legnaro, Italy

${ }^{76}$ Lawrence Berkeley National Laboratory, Berkeley, California, United States

77 Moscow Engineering Physics Institute, Moscow, Russia

${ }^{78}$ Nagasaki Institute of Applied Science, Nagasaki, Japan

${ }^{79}$ Physics Department, National and Kapodistrian University of Athens, Athens, Greece

${ }^{80}$ National Centre for Nuclear Studies, Warsaw, Poland

${ }^{81}$ National Institute for Physics and Nuclear Engineering, Bucharest, Romania

${ }^{82}$ National Institute of Science Education and Research, Bhubaneswar, India

${ }^{83}$ National Research Centre Kurchatov Institute, Moscow, Russia

${ }^{84}$ Niels Bohr Institute, University of Copenhagen, Copenhagen, Denmark

${ }^{85}$ Nikhef, Nationaal instituut voor subatomaire fysica, Amsterdam, Netherlands

${ }^{86}$ Nuclear Physics Group, STFC Daresbury Laboratory, Daresbury, United Kingdom

${ }^{87}$ Nuclear Physics Institute, Academy of Sciences of the Czech Republic, Řež u Prahy, Czech Republic

${ }^{88}$ Oak Ridge National Laboratory, Oak Ridge, Tennessee, United States

${ }^{89}$ Petersburg Nuclear Physics Institute, Gatchina, Russia

${ }^{90}$ Physics Department, Creighton University, Omaha, Nebraska, United States

${ }^{91}$ Physics Department, Panjab University, Chandigarh, India

92 Physics Department, University of Cape Town, Cape Town, South Africa

${ }^{93}$ Physics Department, University of Jammu, Jammu, India

${ }^{94}$ Physics Department, University of Rajasthan, Jaipur, India

95 Physikalisches Institut, Eberhard Karls Universität Tübingen, Tübingen, Germany

${ }^{96}$ Physikalisches Institut, Ruprecht-Karls-Universität Heidelberg, Heidelberg, Germany

${ }^{97}$ Physik Department, Technische Universität München, Munich, Germany

${ }^{98}$ Purdue University, West Lafayette, Indiana, United States

${ }^{99}$ Pusan National University, Pusan, South Korea

${ }^{100}$ Research Division and ExtreMe Matter Institute EMMI, GSI Helmholtzzentrum für Schwerionenforschung GmbH, Darmstadt, Germany

${ }^{101}$ Rudjer Bošković Institute, Zagreb, Croatia

102 Russian Federal Nuclear Center (VNIIEF), Sarov, Russia

${ }^{103}$ Saha Institute of Nuclear Physics, Kolkata, India

${ }^{104}$ School of Physics and Astronomy, University of Birmingham, Birmingham, United Kingdom

105 Sección Física, Departamento de Ciencias, Pontificia Universidad Católica del Perú, Lima, Peru

106 Sezione INFN, Bari, Italy

107 Sezione INFN, Bologna, Italy

${ }^{108}$ Sezione INFN, Cagliari, Italy

${ }^{109}$ Sezione INFN, Catania, Italy

${ }^{110}$ Sezione INFN, Padova, Italy

${ }^{111}$ Sezione INFN, Rome, Italy

${ }^{112}$ Sezione INFN, Trieste, Italy

${ }^{113}$ Sezione INFN, Turin, Italy

${ }^{114}$ SSC IHEP of NRC Kurchatov Institute, Protvino, Russia

115 Stefan Meyer Institut für Subatomare Physik (SMI), Vienna, Austria

116 SUBATECH, Ecole des Mines de Nantes, Université de Nantes, CNRS-IN2P3, Nantes, France

${ }^{117}$ Suranaree University of Technology, Nakhon Ratchasima, Thailand

118 Technical University of Košice, Košice, Slovakia

119 Technical University of Split FESB, Split, Croatia

${ }^{120}$ The Henryk Niewodniczanski Institute of Nuclear Physics, Polish Academy of Sciences, Cracow, Poland

${ }^{121}$ Physics Department, The University of Texas at Austin, Austin, Texas, United States

${ }^{122}$ Universidad Autónoma de Sinaloa, Culiacán, Mexico

${ }^{123}$ Universidade de São Paulo (USP), São Paulo, Brazil

${ }^{124}$ Universidade Estadual de Campinas (UNICAMP), Campinas, Brazil 
${ }^{125}$ Universidade Federal do ABC, Santo Andre, Brazil

${ }^{126}$ University of Houston, Houston, TX, USA

127 University of Jyväskylä, Jyväskylä, Finland

${ }^{128}$ University of Liverpool, Liverpool, United Kingdom

${ }^{129}$ University of Tennessee, Knoxville, Tennessee, United States

${ }^{130}$ University of the Witwatersrand, Johannesburg, South Africa

${ }^{131}$ University of Tokyo, Tokyo, Japan

${ }^{132}$ University of Tsukuba, Tsukuba, Japan

${ }^{133}$ University of Zagreb, Zagreb, Croatia

${ }^{134}$ Université de Lyon, Université Lyon 1, CNRS/IN2P3, IPN-Lyon, Villeurbanne, Lyon, France

135 Università di Brescia, Brescia, Italy

${ }^{136}$ V. Fock Institute for Physics, St. Petersburg State University, St. Petersburg, Russia

137 Variable Energy Cyclotron Centre, Kolkata, India

${ }^{138}$ Warsaw University of Technology, Warsaw, Poland

${ }^{139}$ Wayne State University, Detroit, Michigan, United States

140 Wigner Research Centre for Physics, Hungarian Academy of Sciences, Budapest, Hungary

${ }^{141}$ Yale University, New Haven, Connecticut, United States

142 Yonsei University, Seoul, South Korea

${ }^{143}$ Zentrum für Technologietransfer und Telekommunikation (ZTT), Fachhochschule Worms, Worms, Germany

${ }^{a}$ Deceased

${ }^{b}$ Also at: Georgia State University, Atlanta, Georgia, United States

${ }^{c}$ Also at: Also at Department of Applied Physics, Aligarh Muslim University, Aligarh, India

d Also at: M.V. Lomonosov Moscow State University, D.V. Skobeltsyn Institute of Nuclear, Physics, Moscow, Russia 